\title{
Experimental Radionuclide Therapy of HER2-Expressing Xenografts Using Two-Step Targeting Nuclisome Particles
}

\author{
Lars Gedda $^{1}$, Amelie Fondell ${ }^{1}$, Hans Lundqvist ${ }^{1}$, John W. Park ${ }^{2}$, and Katarina Edwards ${ }^{3,4}$ \\ ${ }^{1}$ Biomedical Radiation Sciences, Rudbeck Laboratory, Uppsala University, Uppsala, Sweden; ${ }^{2}$ Department of Medicine, Division of \\ Haematology-Oncology, Cancer Research Institute, University of California San Francisco, San Francisco, California; ${ }^{3}$ Department \\ of Chemistry, Uppsala University, Uppsala, Sweden; and ${ }^{4}$ FRIAS, School of Soft Matter Research, University of Freiburg, Freiburg, \\ Germany
}

The therapeutic potential of Auger-electron emitting radionuclides is strongly dependent on their close vicinity to DNA, since the energy deposition is mainly localized within a few cubic nanometers around the site of decay. Thus, apart from specificity, successful tumor therapy relies on a nuclear delivery strategy. We recently presented a two-step targeting strategy to transport Auger-electron-emitting radionuclides into the cell nucleus by means of nuclide-filled liposomes (Nuclisome particles), that is, polyethylene glycol-stabilized, tumor-celltargeting liposomes loaded with ${ }^{125}$ I-labeled anthracyclines. In the present study, the survival of mice intraperitoneally inoculated with human HER2-expressing SKOV-3 tumor cells and treated with HER2-targeting Nuclisome particles was studied. Methods: BALB/c nu/nu mice were inoculated with $10^{7}$ SKOV3 cells intraperitoneally and thereafter directly injected with Nuclisome particles with increasing specific radioactivity. Groups of $10-12$ mice were treated with $0.01 \mathrm{MBq} /$ mouse up to $2 \mathrm{MBq} /$ mouse, and survival was monitored and compared with that in control groups $(n=33)$. Organs were analyzed for HER2 expression and radiotoxic effects histologically. Absorbed doses were estimated using dose factors from the online Radiation Dose Assessment Resource model. Results: The results showed a clear correlation between administered radioactive dose and survival. No such dose-dependent survival was observed for mice treated with Nuclisome particles lacking HER2-targeting ability. With HER2-targeting Nuclisome particles, a significant increase in survival, compared with that of untreated control mice, could already be seen at an administered activity of $0.1 \mathrm{MBq} / \mathrm{mouse}(P=0.0301)$. At the highest activity administered, $2 \mathrm{MBq} /$ mouse $(P<0.0001), 70 \%$ of the mice survived the study and most were tumor-free. Neither macroscopic nor microscopic radiotoxic side effects were observed. Dosimetric calculations, assuming nonreceptor targeting, revealed that the radioactive doses to normal tissues were low. Conclusion: Taken together the results show that with successful targeting to the tumor-cell nucleus it is possible to obtain a therapeutic effect from Auger-electron-emitting radionuclides administered at radioactive doses low enough to spare normal tissue from radiotoxic side effects.

\footnotetext{
Received Aug. 12, 2011; revision accepted Oct. 25, 2011.

For correspondence contact: Lars Gedda, Department of Radiology, Oncology and Radiation Sciences, Unit of Biomedical Radiation Sciences, Rudbeck Laboratory, Uppsala University, 75185 Uppsala, Sweden.

E-mail: Lars.Gedda@bms.uu.se

Published online Feb. 8, 2012.

COPYRIGHT (c) 2012 by the Society of Nuclear Medicine, Inc.
}

Key Words: HER2; liposome; intercalator; Auger; tumortargeting

J Nucl Med 2012; 53:480-487

DOI: 10.2967/jnumed.111.096891

$\mathbf{T}$ he potential use of short-range, low-energy Augerelectron-emitting radionuclides for tumor therapy was first suggested some $40 \mathrm{y}$ ago when it was realized that these nuclides could induce severe damage to cellular DNA (1). It was observed that, as a consequence of the multiple electron cascades during decay, complex double-strand breaks were produced when the nuclides were introduced within DNA. Since most of the energy deposition from these electrons is highly localized in a small volume, it early became clear that their therapeutic value was limited to situations in which positioning of the nuclides within or close to DNA was achievable. Several successful attempts were made to accomplish DNA association of the radionuclides (2), but at the time no functional tumor-cell-specific delivery system was at hand and therefore the approach had no true success.

With the advent of innovative delivery concepts, the field has been revitalized during the last decade, and several approaches to deliver Auger-electron-emitting radionuclides specifically to tumor-cell nuclei have recently been suggested and tested (3-6). The existing strategies are based mainly on the use of nuclear localization sequences to transport radiolabeled peptides or proteins from cytoplasmatic compartments to the nucleus. We have taken a different approach (7) and use a two-step targeting principle to achieve DNAtargeted delivery of the radionuclides. The concept is based on the use of targeting liposomes loaded with substantial quantities of a radiolabeled DNA-intercalating compound referred to as ${ }^{125}$ I-Comp1 (8). This approach enables, in a first step, specific delivery of large amounts of Auger-electronemitting radionuclides to the tumor cells and facilitates, in a second step, localization of the internalized radionuclides close to DNA in the cell nucleus. By this strategy, we aim to maximize the so-called Auger effect-the very local effect of high electron density causing complex DNA damage (9). We 
call our nuclide-filled liposomes Nuclisome particles and have in previous studies demonstrated a specific and considerable growth inhibitory effect of Nuclisome particles on cultured human tumor cells $(7,10)$.

In this study, we present the results of a dose-escalating investigation focused on survival of tumor-bearing mice treated with HER2-targeting and nontargeting liposomes encapsulating the Auger-electron-emitting compound ${ }^{125} \mathrm{I}$-Comp1. Further, we report data from dosimetric calculations and from morphologic evaluations of critical normal organs.

\section{MATERIALS AND METHODS}

\section{Chemicals}

1,2-distearoyl-sn-glycero-3-phosphatidylcholine and 1,2-distearoyl-sn-glycero-3-phosphatidylethanol-amine- $N$-[methoxy(polyethylene glycol)-2000] were purchased from Avanti Polar Lipids Inc. Citric acid, $N$-[2-hydroxyethyl]piperazine- $N^{\prime}$-[2-ethanesulfonic acid] (HEPES), cholesterol, and chloramine-T were purchased from Sigma-Aldrich. Sephadex G-25 columns and Sepharose CL-4B were purchased from Amersham Biosciences and ${ }^{125}$ I from Perkin Elmer. Other chemicals were purchased from common sources. Amino-benzyl derivatives of the anthracycline daunorubicin were synthesized as their formate salts as described previously (11). Anthracyclines are known for their ability to intercalate with the B-form of the DNA helix through GC site-specific interactions (12). The biologic characterization of the compound, referred to as Comp1, showed the compound's ability to bind DNA, displace the anthracycline doxorubicin, and render DNA damage during radioiodine decay (8). Comp1 was radiolabeled using the chloramine-T method, purified, and encapsulated in liposomes as described previously (10).

\section{Targeting Liposomes}

F5 is a single-chain variable fragment directed against HER2 (13). In brief, $12 \mu \mathrm{g}$ of F5-PEG ${ }_{2000}$-DSPE $/ \mu$ mol total lipid of ${ }^{125} \mathrm{I}-\mathrm{Comp} 1-$ loaded liposomes was mixed at a temperature of $60^{\circ} \mathrm{C}$ for $1 \mathrm{~h}$. Unincorporated $\mathrm{F} 5-\mathrm{PEG}_{2000}$-DSPE was removed from the liposomes on a Sepharose CL-4B column with HEPES buffered saline (pH 7.4) as the mobile phase (7). All fractions were measured with a handheld scintillation detector, and fractions corresponding to F5-liposome- ${ }^{125}$ IComp1 were pooled.

\section{Cell Culture}

SKOV-3 (human ovarian adenocarcinoma, ATCC HTB-77) and SKBR-3 (breast adenocarcinoma, ATCC HTB-30) were obtained from American Type Culture Collection. Cells overexpressing HER2 were grown in Nunclone $75 \mathrm{~cm}^{2}$ cell culture flasks (Nunc) as monolayer cultures using McCoy (SKOV-3) and RPMI 1640 medium (SKBR-3) supplemented with $10 \%$ fetal calf serum, glutamine (2 $\mathrm{mM})$, streptomycin $(100 \mu \mathrm{g} / \mathrm{mL})$, and penicillin $(100 \mathrm{IU} / \mathrm{mL})$ (Biochrom AG) at a temperature of $37^{\circ} \mathrm{C}$ in humidified air $\left(5 \% \mathrm{CO}_{2}\right)$.

\section{Tumor Model}

Female BALB/c nu/nu mice (Taconic Mollegaard \& Bomholtgaard) were used, and before study the mice were allowed to adapt for at least $7 \mathrm{~d}$. During the whole study, the mice were kept in a controlled environment and fed ad libitum. All experiments were approved by the Regional Ethics Committee for Animal Research.

To find a good model for intraperitoneal HER2 targeting of liposomes, we analyzed the tumor take of the HER2-expressing human tumor cell lines SKOV3 and SKBR3. Mice were in- traperitoneally inoculated with $10^{6}-10^{7}$ cells (4 mice per group). Both trypsinized and scraped cells were analyzed. During tumor growth, the mice were monitored daily and weighed 3 times a week. After 4 and $8 \mathrm{wk}$, the mice were killed and tumor take was scored.

For the biodistribution study, injected tumor cells were allowed to grow until they reached solid tumors (8 wk). This enhanced the examination of the targeting ability of liposomes. For the tumor treatment, no growth was permitted before injection of liposomes. This was to allow targeting of single tumor cells rather than solid tumors.

\section{Biodistribution and Internal Dosimetry}

${ }^{125}$ I decays with electron capture and emit several low-energy Auger and conversion electrons. There is also a substantial amount of low-energy $x$-rays and $\gamma$-radiation emitted. Of the total energy released in the decay, $16.6 \mathrm{keV}$ is emitted as charged particles and 41.6 $\mathrm{keV}$ is emitted as photons. Because of the relatively high photon contribution, a substantial part of the absorbed dose in humans is due to cross fire, that is, photons from an organ irradiating neighboring organs. However, in mice of about $20 \mathrm{~g}$ the cross fire effect is limited since a large part of the photon energy will leave the body (14). For a structure of $1 \mathrm{~g}, 21 \mathrm{keV}$ are absorbed. This is just above the charged particle energy. Further, only $25 \mathrm{keV}$ are absorbed in a spheric weight of $10 \mathrm{~g}$, meaning that the cross fire effect is just slowly increasing. Therefore cross fire was neglected in the calculation of organ doses.

To make a rough estimate of absorbed dose in the mice, we used previous pharmacokinetic data (10), presented as standardized uptake values (SUVs). In pharmacokinetic studies, usually the interest is in presenting the uptake of the molecule, and the organ activities are then corrected for decay. When used for internal dosimetric calculations, the organ values noted as $\mathrm{SUV}^{*}$ were not corrected for decay.

To define SUV*, the organ activity (OA) was divided by the organ weight $(\mathrm{OW})$. This ratio was then divided by the administered activity (AA)/animal weight (AW). We can then write

$$
\mathrm{OA}=\mathrm{OW} \times \mathrm{AA} / \mathrm{AW} \times \mathrm{SUV}^{*} .
$$

Eq. 1

Time integrating OA will give the total number of decays. By multiplying this value by a dose factor (DF) giving dose/decays, we can calculate the absorbed dose.

The SUVs* from spleen, liver, kidneys, and blood were integrated from 0 to $48 \mathrm{~h}$ using the histogram method. From the 2 last time values, an exponential slope was calculated and used to extrapolate the number of decays after $48 \mathrm{~h}$. The obtained value (ISUV*) has the dimension time. The organ dose (OD) can then be given as

$$
\mathrm{OD}=\mathrm{DF} \times \mathrm{OW} \times \mathrm{AA} / \mathrm{AW} \times \mathrm{ISUV}^{*} . \quad \text { Eq. } 2
$$

We used a sphere model in the online Radiation Dose Assessment Resource model (14) to obtain the dose factor converting total number of decays to absorbed dose. The product $\mathrm{DF} \times \mathrm{OW}$ has the dimension absorbed energy per decay and varies little (1-10 g) (14). A value of $0.0035 \mathrm{mGy} \times \mathrm{g} / 1$ million decays was used in the following calculations (14). The animal weights were set to $20 \mathrm{~g}$.

\section{Tumor Treatment}

Before animal injections, prepared liposomes (targeting and nontargeting) were inspected for size and shape and the precipitate of loaded compound using cryogenic transmission electron microscopy. The experimental setting was described previously (7). Further, 
radioactive concentrations of all prepared liposome solutions were measured using an ion chamber (VDC-PS; Studsvik Instruments).

Nuclisome particles for tumor treatment were administered intraperitoneally directly after tumor cell injection. Groups of mice were randomly selected at 3 successive dose-escalating studies. At a first study, 0.01 MBq of HER2-targeting liposomes $(n=12), 0.1 \mathrm{MBq}$ of HER2-targeting liposomes $(n=12), 0.1$ $\mathrm{MBq}$ of nontargeting liposomes $(n=12)$, and controls (HEPES buffered saline, $n=12$ ) were investigated. In a second study, 0.5 MBq of HER2-targeting liposomes $(n=10), 0.5 \mathrm{MBq}$ of nontargeting liposomes $(n=10)$, and controls (HEPES buffered saline, $n=10$ ) were investigated. In a third study, $2 \times 0.5 \mathrm{MBq}$ of HER2-targeting liposomes $(n=12), 2 \times 0.5 \mathrm{MBq}$ of nontargeting liposomes $(n=12), 2 \mathrm{MBq}$ of HER2-targeting liposomes $(n=$ $12), 2 \mathrm{MBq}$ of nontargeting liposomes $(n=12)$, and controls (HEPES buffered saline, $n=11$ ) were investigated. All animals except controls received $0.2 \mathrm{~mL}$ of liposomes $(0.25 \mu \mathrm{mol})$ in HEPES buffered saline. Only the specific activity of ${ }^{125}$ I-Comp1 was varied; the total concentration of ${ }^{125}$ I-Comp1 was the same in all treatments. Controls received HEPES buffered saline only.

After injection, the key was not revealed for persons monitoring the animal's state of health, keeping the identity of the groups secret until after the data analysis. The mice were monitored each day for radiotoxic symptoms or behavior disorders and were weighed 3 times per week. The animals were monitored until their general condition declined or body weight rapidly increased or decreased, and at that time they were killed and dissected. Tumors, spleen, and liver were dissected for analysis of HER2 expression and possible morphologic transformation. Survival curve plots and Kaplan-Meier analysis were done using Prism 5 (GraphPad Software). The mice removed from the study for reasons other than having reached the endpoint (killed due to tumor burden) were censored. The study was, for the remaining animals, finished after $160 \mathrm{~d}$, at which time they were censored.

\section{Histology}

Tumors, spleen, and liver were fixed in formalin (4\% phosphatebuffered formaldehyde), dehydrated, and embedded in paraffin. Histologic sections of $4 \mu \mathrm{m}$ were cut and dried on microscopic slides for $1 \mathrm{~h}$ at $50^{\circ} \mathrm{C}$. The sections were freed of paraffin and rehydrated. The slides were stained with hematoxylin and eosin for toxicologic analysis, and some slides were stained for HER2 expression. For HER2 expression analysis, slides were treated with hydrogen peroxide-doped phosphate-buffered saline for $10 \mathrm{~min}$ to quench endogenous peroxidase. Retrieval was performed by pressure boiling the samples in citrate buffer for $7 \mathrm{~min}$. The samples were stained for HER2 with the A0485 antibody (DAKO). Antibody dilution according to the manufacturer was applied (1:350), and the samples were incubated at $4{ }^{\circ} \mathrm{C}$ overnight. After rinsing, the slides were treated with EnVision antibody (DAKO) and incubated for $45 \mathrm{~min}$ at room temperature. The samples were rinsed again and then developed using fuchsin substrate (DAKO) for $15 \mathrm{~min}$. The slides were mounted and examined for morphologic damage by a trained pathologist.

\section{Statistical Analysis}

In Kaplan-Meyer survival plots, significance was analyzed using the Log-rank test (Mantel-Cox).

\section{RESULTS}

\section{Characterization of Liposomes}

Liposome and Nuclisome particle preparations were routinely analyzed by cryogenic transmission electron microscopy to confirm that ${ }^{125}$ I-Comp1 was present inside the liposomes in precipitated form and to ensure that the liposomes were mainly unilamellar and had the expected size of about $100 \mathrm{~nm}$. All preparations used in the present study displayed a general appearance similar to that observed and reported in the previous cell therapy study (10).

\section{Tumor Model}

Neither the amount of injected cells nor the time for tumor establishment affected tumor take for SKBR3 cells. Generally, tumor take was low and, at a later time, even nonexistent. In contrast, tumors were easily established with SKOV3 cells. With injection of $10^{7}$ cells, tumors were already present within $4 \mathrm{wk}$, and as judged after $8 \mathrm{wk}$, the establishment was independent of preparation method (Table 1). The tumors were solid and present mainly at the lymph node underneath liver. There were no signs of microtumors on the peritoneum or colon, and no ascitic fluid was noticed.

\section{Biodistribution and Internal Dosimetry}

A previous biodistribution study (10) presented the pharmacokinetics of the studied liposomes. Data for spleen, liver, kidneys, blood, and tumors were extracted (Table 2) and used for calculation of tumor-to-organ ratios and for dosimetric calculations.

Tumor-to-organ values for targeting liposomes were above 1 for all organs except spleen, whereas for nontargeting liposomes these values were low. Especially, tumor-to-blood values increased from about 0.7 to almost 100 at $8 \mathrm{~h}$, when a change was made from nontargeting to targeting liposomes. The only 2 organs that, besides tumor, substantially accumulated ${ }^{125} \mathrm{I}$ from targeting liposomes were liver and spleen.

The calculated organ doses per megabecquerel are given in Table 3. These doses do not take into account the Auger effect that occurs when ${ }^{125} \mathrm{I}$ is positioned close to DNA. Since it is anticipated that two-step targeting is nonfunctional in organs not expressing HER2, the calculated doses are relevant as a measure of normal-organ doses but not tumor dose. The highest normal-organ dose is given to

TABLE 1

Tumor Take of Cells Injected Intraperitoneally

\begin{tabular}{|c|c|c|c|}
\hline \multirow{3}{*}{$\begin{array}{l}\text { Cell type } \\
\text { and number }\end{array}$} & \multicolumn{3}{|c|}{ Tumor uptake } \\
\hline & \multirow[b]{2}{*}{4 wk (trypsinized) } & \multicolumn{2}{|c|}{$8 w k$} \\
\hline & & Trypsinized & Scraped \\
\hline $10^{6}$ SKBR3 & + & - & NA \\
\hline $10^{7}$ SKBR3 & + & - & - \\
\hline $10^{6}$ SKOV3 & + & ++ & NA \\
\hline $10^{7}$ SKOV3 & +++ & +++ & +++ \\
\hline \multicolumn{4}{|c|}{$\begin{array}{l}-=0 \%-24 \% ;+=25 \%-49 \% ;++=50 \%-74 \% ;+++= \\
75 \%-100 \% ; N A=\text { not analyzed. }\end{array}$} \\
\hline
\end{tabular}


TABLE 2

Extracted SUV from Biodistribution in Intraperitoneal Xenografts (10)

\begin{tabular}{|c|c|c|c|c|c|c|}
\hline \multirow[b]{2}{*}{ Tissue type } & \multicolumn{6}{|c|}{ Time } \\
\hline & $10 \mathrm{~min}$ & $2 \mathrm{~h}$ & $4 \mathrm{~h}$ & $8 \mathrm{~h}$ & $24 \mathrm{~h}$ & $48 \mathrm{~h}$ \\
\hline \multicolumn{7}{|c|}{ HER2-targeting } \\
\hline Blood & $0.13 \pm 0.19$ & $0.54 \pm 1.20$ & $0.08 \pm 0.03$ & $0.05 \pm 0.01$ & $0.02 \pm 0.01$ & $0.01 \pm 0.01$ \\
\hline Spleen & $0.42 \pm 0.15$ & $8.35 \pm 10.09$ & $6.18 \pm 6.48$ & $12.81 \pm 4.32$ & $3.01 \pm 1.79$ & $0.61 \pm 0.36$ \\
\hline Liver & $0.19 \pm 0.02$ & $2.73 \pm 2.46$ & $2.50 \pm 1.63$ & $2.42 \pm 1.01$ & $0.24 \pm 0.05$ & $0.05 \pm 0.05$ \\
\hline Kidneys & $0.21 \pm 0.06$ & $0.46 \pm 0.37$ & $0.69 \pm 0.32$ & $0.53 \pm 0.03$ & $0.21 \pm 0.08$ & $0.07 \pm 0.05$ \\
\hline Tumor & $1.03 \pm 0.37$ & $2.11 \pm 1.92$ & $2.95 \pm 2.09$ & $3.56 \pm 2.85$ & $0.55 \pm 0.31$ & $0.29 \pm 0.16$ \\
\hline \multicolumn{7}{|l|}{ Nontargeting } \\
\hline Blood & $0.39 \pm 0.54$ & $2.99 \pm 1.28$ & $2.21 \pm 0.92$ & $1.56 \pm 0.69$ & $0.08 \pm 0.10$ & $0.00 \pm 0.00$ \\
\hline Spleen & $0.24 \pm 0.20$ & $2.80 \pm 2.06$ & $2.51 \pm 0.99$ & $2.88 \pm 0.40$ & $0.67 \pm 0.64$ & $0.12 \pm 0.05$ \\
\hline Liver & $0.12 \pm 0.08$ & $0.895 \pm 0.30$ & $1.08 \pm 0.52$ & $1.56 \pm 0.31$ & $0.17 \pm 0.08$ & $0.02 \pm 0.00$ \\
\hline Kidneys & $0.22 \pm 0.22$ & $0.91 \pm 0.56$ & $1.15 \pm 0.30$ & $1.20 \pm 0.14$ & $0.31 \pm 0.22$ & $0.02 \pm 0.01$ \\
\hline Tumor & $0.71 \pm 0.58$ & $0.62 \pm 0.45$ & $1.02 \pm 0.64$ & $0.94 \pm 0.11$ & $0.29 \pm 0.09$ & $0.11 \pm 0.09$ \\
\hline
\end{tabular}

Errors are maximal variation $(n=5)$.

spleen, with $150 \mathrm{mGy}$ per administered megabecquerel. However, already at $0.1 \mathrm{MBq}$ there was a significant effect on survival, giving a dose to spleen of only about $15 \mathrm{mGy}$.

\section{Tumor Treatment}

Neither radiotoxic observations, which are expected to appear within the first 2-3 wk, nor chemotoxic observations were made during the treatment. The general health condition did not change for any of the animals within the different groups until about $50 \mathrm{~d}$ after inoculation. As shown in the Kaplan-Meier plots in Figure 1, the mice were then starting to be affected by the tumor burden. Mice that by ocular inspection could be judged as affected — by a swollen or blue abdomen, decreased overall health, or a rapid weight change - were killed. The endpoint criterion for the KaplanMeier analysis was "killed by tumor burden." The endpoint was reached by 126 of 148 mice from all 3 studies.

Figure 1A shows that neither the group receiving HER2targeting liposomes with $0.01 \mathrm{MBq} /$ mouse nor the group receiving nontargeting liposomes with $0.1 \mathrm{MBq} /$ mouse differed from the control group that received HEPES buffered saline only. In the group receiving HER2-targeting lipo-

TABLE 3

Doses to Normal Organs as Function of Administered Radioactivity

\begin{tabular}{lcc}
\hline & \multicolumn{2}{c}{ mGy/MBq administered radioactivity } \\
\cline { 2 - 3 } Tissue type & HER2-targeting & Nontargeting \\
\hline Blood & 1.8 & 18.7 \\
Spleen & 150.0 & 36.6 \\
Liver & 27.1 & 15.4 \\
Kidneys & 9.4 & 15.0
\end{tabular}

Mean doses are presented under assumption that distribution of activity in therapy study follows biodistribution of HER2targeting and nontargeting liposomes presented in Table 2. somes with $0.1 \mathrm{MBq} /$ mouse, 3 mice had not reached the endpoint by the end of study ( $160 \mathrm{~d})$. Of these mice, 2 were tumor-free. One mouse in the control group was found dead after $24 \mathrm{~d}$; however, on dissection no tumor could be seen. All these mice were censored.

As shown in Figure 1B, a greater difference between the control group and the group treated with HER2-targeting liposomes could be observed when the dose was increased to $0.5 \mathrm{MBq} /$ mouse. Two mice had not reached the endpoint by the end of study and were censored. Both were found to be tumor-free. The survival of mice receiving nontargeting liposomes with $0.5 \mathrm{MBq} / \mathrm{mouse}$ did not differ from that of controls, although 1 mouse lived almost $160 \mathrm{~d}$ before reaching the endpoint. Changing the route of administration to repeated injections of $0.5 \mathrm{MBq} /$ mouse with $7 \mathrm{~d}$ between the injections increased the survival of mice treated with HER2targeting liposomes (Fig. 1C). Four mice had not reached the endpoint by the end of the study and were censored. Three of the 4 mice were found to be tumor-free. One mouse was killed at day 70 but was found not to have reached the endpoint and was therefore censored. Survival in the group receiving nontargeting liposomes with $2 \times 0.5 \mathrm{MBq} /$ mouse did not differ from that in the control group.

A single dose of $2 \mathrm{MBq} /$ mouse was found to be the most effective in terms of prolonging survival, as seen in Figure 1C. In the group treated with HER2-targeting liposomes, $70 \%$ of the mice were still alive $160 \mathrm{~d}$ after treatment, whereas in the control group and the group treated with nontargeting liposomes, all mice were killed because of tumor burden within 85 and $140 \mathrm{~d}$, respectively. Two mice in the group treated with HER2-targeting liposomes were killed at days 91 and 119, respectively, and 2 mice in the group treated with nontargeting liposomes were killed at days 75 and 107, respectively. These 4 mice were found not to have reached the endpoint. An additional 7 mice had not reached the endpoint by the end of the study. Four of these 7 mice were found to be tumor-free. All these mice were censored. 


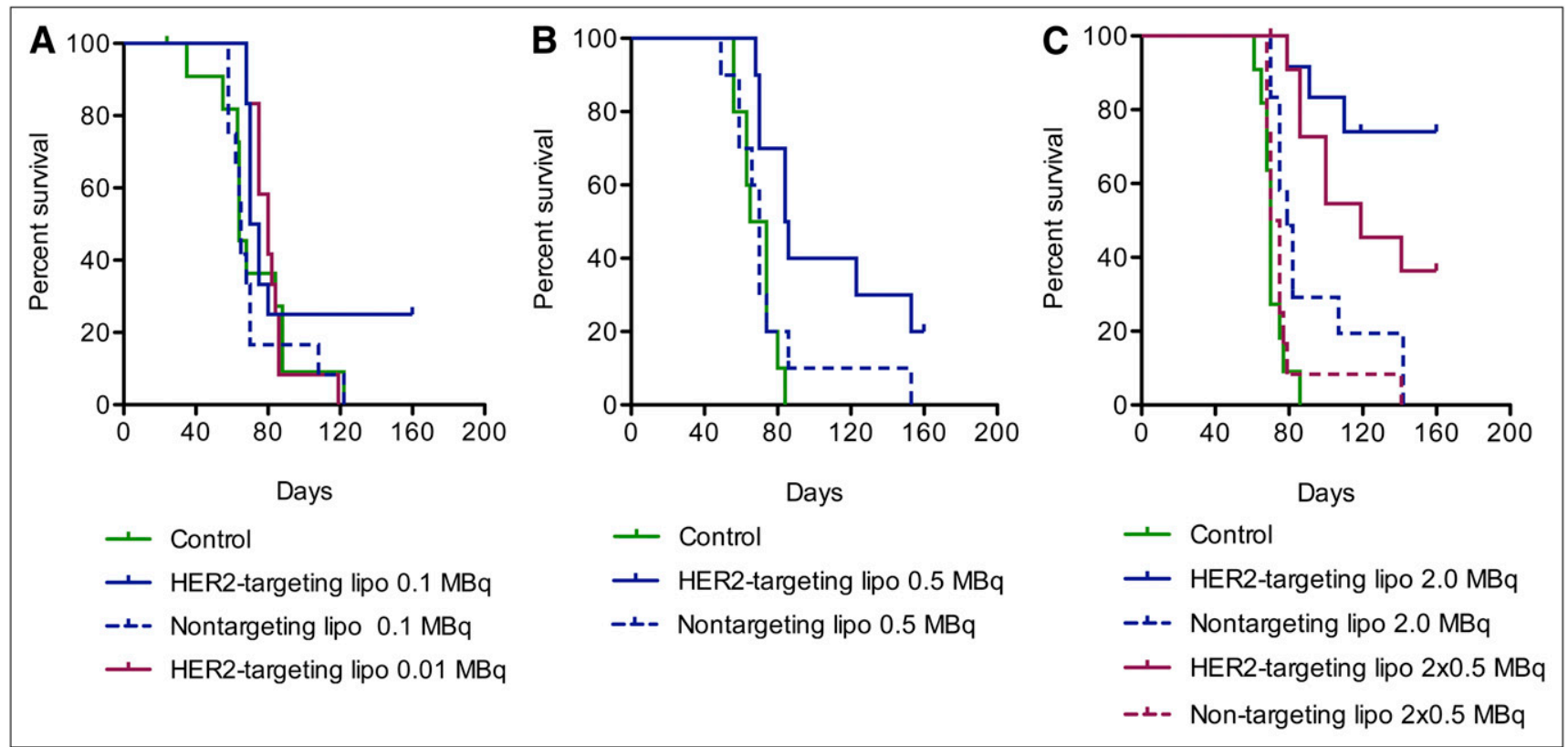

FIGURE 1. Kaplan-Meier plot showing survival of mice with SKOV-3 intraperitoneal xenografts. (A) Mice treated with $0.01 \mathrm{MBq}$ per mouse $(n=12)$, with $0.1 \mathrm{MBq}$ of HER2-targeting liposomes per mouse $(n=12)$, and with $0.1 \mathrm{MBq}$ of nontargeting liposomes per mouse $(n=12)$ (control [HEPES buffered saline] $n=12$ ). (B) Mice treated with $0.5 \mathrm{MBq}$ of HER2-targeting liposomes per mouse $(n=10)$ and with $0.5 \mathrm{MBq}$ of nontargeting liposomes per mouse $(n=10)$ (control [HEPES buffered saline] $n=10)$. (C) Mice treated with $2 \times 0.5 \mathrm{MBq}$ per mouse $(n=$ 12), with $2 \mathrm{MBq}$ of HER2-targeting liposomes per mouse $(n=12)$, with $2 \times 0.5 \mathrm{MBq}$ per mouse $(n=12)$, and with $2 \mathrm{MBq}$ of nontargeting liposomes per mouse $(n=12)$ (control [HEPES buffered saline], $n=11$ ).

Figure 2 shows pooled data from all dose-escalating studies. With pooled data, the control group is stronger and the analysis of significance is better performed. In the control group, all mice $(n=33)$ except one reached the endpoint. The sole mouse not reaching the endpoint was censored since no tumor burden was revealed.

From the compilation shown in Figure 2A, it is evident that treatment with nontargeting liposomes at doses corre-
FIGURE 2. Kaplan-Meier plot showing survival of mice with SKOV-3 intraperitoneal xenografts pooled from the 3 dose-escalating studies in Figure 1 (control [HEPES buffered saline], $n=33$ ). (A) Mice treated with nontargeting liposomes. (B) Mice treated with HER2targeting liposomes.

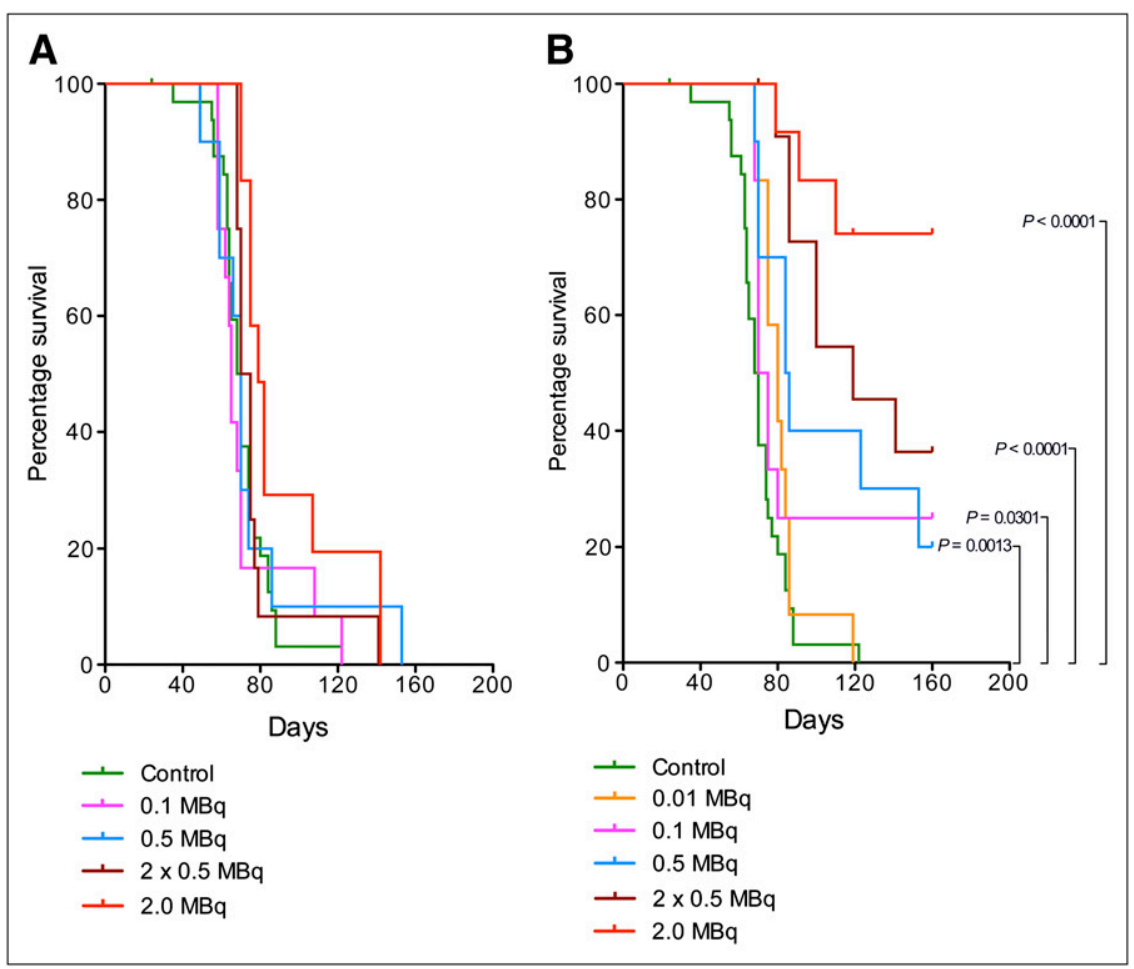


sponding to $0.1,0.5$, or $2 \times 0.5 \mathrm{MBq} /$ mouse did not increase survival of the mice $(P=0.3041-0.8685)$. Treatment with nontargeting liposomes at a dose corresponding to $2 \mathrm{MBq} /$ mouse resulted, however, in a significant increase in survival $(P=0.0079)$. Median survival in all the groups treated with nontargeting liposomes was similar to that in the control group (Table 4).

The data presented in Figure 2B show that for mice treated with HER2-targeting liposomes there was a clear and positive relationship between dose and survival. Mice treated with the lowest dose $(0.01 \mathrm{MBq} / \mathrm{mouse})$ did not significantly differ from controls $(P=0.0958)$, but at $0.1 \mathrm{MBq} /$ mouse there already was a significant increase in survival $(P=0.0301)$. Although $0.5 \mathrm{MB} /$ mouse gave a further increase in survival $(P=$ $0.0013)$, a repeated injection $7 \mathrm{~d}$ after the first treatment prolonged survival much more $(P<0.0001)$. Table 4 also shows that the median survival was almost doubled compared with that of controls when the mice were treated with repeated injections of $0.5 \mathrm{MBq} / \mathrm{mouse}$. From Figure $2 \mathrm{~B}$ it is apparent, however, that the greatest impact on survival $(P<0.0001)$ was obtained for mice treated with a single dose corresponding to $2 \mathrm{MBq} /$ mouse. No median survival could be determined for this group since so few mice reached the endpoint.

\section{Histology}

As analyzed by immunohistochemistry, the solid tumors appearing after intraperitoneal injection of SKOV3 cells were HER2-positive (Fig. 3). Spleen and liver stained negatively for HER2. Noticeable is that during the treatment study the tumors in 2 animals showed down-regulated HER2 expression and partly differentiated morphology. Those 2 mice were both within the control group and-like the other mice in the control group_-did eventually reach the endpoint. Therefore, the deviation in cell behavior was not believed to have affected the outcome of the study.

Spleen and liver from controls and mice treated with HER2targeting liposomes at doses corresponding to $0.5 \mathrm{MBq} / \mathrm{mouse}$, $2 \times 0.5 \mathrm{MBq} / \mathrm{mouse}$, and $2 \mathrm{MBq} /$ mouse were inspected in a masked manner by a pathologist for morphologic damage. In general, liver showed intact parenchyma, and no signs of necrosis, inflammation, or increased fattiness were observed.

TABLE 4

Median Survival of Mice Treated with HER2-Targeting and Nontargeting Liposomes

\begin{tabular}{lcc}
\hline & \multicolumn{2}{c}{ Median survival } \\
\cline { 2 - 3 } MBq/mouse & HER2-targeting & Nontargeting \\
\hline Control & 69 & 69 \\
0.01 & 72.5 & NA \\
0.1 & 85 & 65 \\
0.5 & 119 & 70 \\
$2 \times 0.5$ & $>160$ & 72.5 \\
2 & & 79 \\
& & \\
\hline NA $=$ not analyzed. & & \\
\hline
\end{tabular}

In 2 of the mice, tumors contributing to some inflammation and necrosis were revealed within the liver. Both mice were found to be tumor-positive after the end of the study (one from $2 \mathrm{MBq} /$ mouse and the other from $2 \times 0.5 \mathrm{MBq} / \mathrm{mouse})$. In general, spleen showed a normal blood status and no signs of necrosis. In 1 spleen, some necrosis with granulocytes was observed but the blood status was otherwise normal. This mouse was found to be tumor-free after the end of the study ( $2 \mathrm{MBq} / \mathrm{mouse}$ ), and in this case the spleen could have received radiotoxic or cytotoxic damage from the treatment. However, this single spleen was the only one that deviated from normal.

\section{DISCUSSION}

We have previously shown that the radiolabeled DNA binder, ${ }^{125} \mathrm{I}-\mathrm{Comp} 1$, used in this study can produce severe DNA damage (8). Further, when the compound is loaded into EGFR- or HER2-targeting liposomes, receptor-specific uptake and severe growth disturbances of EGFR- or HER2-expressing cultured human tumor cells are observed $(7,10)$. Importantly, a significant cell therapeutic effect is observed at concentrations for which doxorubicin-loaded liposomes or nontargeting liposomes loaded with ${ }^{125} \mathrm{I}$-Comp1 have no effect. The aims of this study were to investigate how the radioactive dose delivered via Nuclisome particles correlated to the survival of xenograft mice and to estimate the radioactive dose distributed to critical normal organs.

The big differences in tumor-to-organ ratios, especially tumor-to-blood ratios, between HER2-targeting and nontargeting liposomes constitutes a strong indication of receptor-specific uptake of the targeted liposomes. Successful targeting of tumor cells in the abdominal cavity presumably decreases the outflow of targeting liposomes into the blood circulation. A subsequent effect would be that a higher tumor-to-blood ratio is achieved for targeting than nontargeting liposomes. Further, the decreased amount of radioactivity in blood after distribution of targeting liposomes is an advantage because of the consequent minimization of the risk that red bone marrow will become a critical organ. Importantly, the use of Auger-emitting radionuclides with a low photon contribution will further lower this risk for both HER2-targeting and nontargeting liposomes. From the dosimetric calculation, it can be concluded that the doses to bone marrow are low in all cases. Even for the case resulting in the highest administered dose, that is, when nontargeting liposomes are used to deliver radioactivity corresponding to $2 \mathrm{MBq} /$ mouse, the dose to blood was as low as $37.5 \mathrm{mGy}$. For targeting liposomes, that is, Nuclisome particles, the dose to blood was reduced to $3.6 \mathrm{mGy}$ for mice treated with $2 \mathrm{MBq}$ and to merely $0.2 \mathrm{mGy}$ (still a significant effect on survival) for mice treated with $0.1 \mathrm{MBq}$.

The fact that spleen and liver were the 2 organs that, besides tumor, accumulated ${ }^{125}$ I from targeting liposomes was not unexpected, since these organs handle foreign particles and tend to entrap liposomes. Both liver and spleen have filtering structures that can trap larger particles, and 
FIGURE 3. Histologic samples of organs: immunohistochemical staining of HER2 from control mice and sample of organs used for morphologic evaluation from mice in tumor treatment assay. $\mathrm{H} \& \mathrm{E}=$ hematoxylin and eosin.

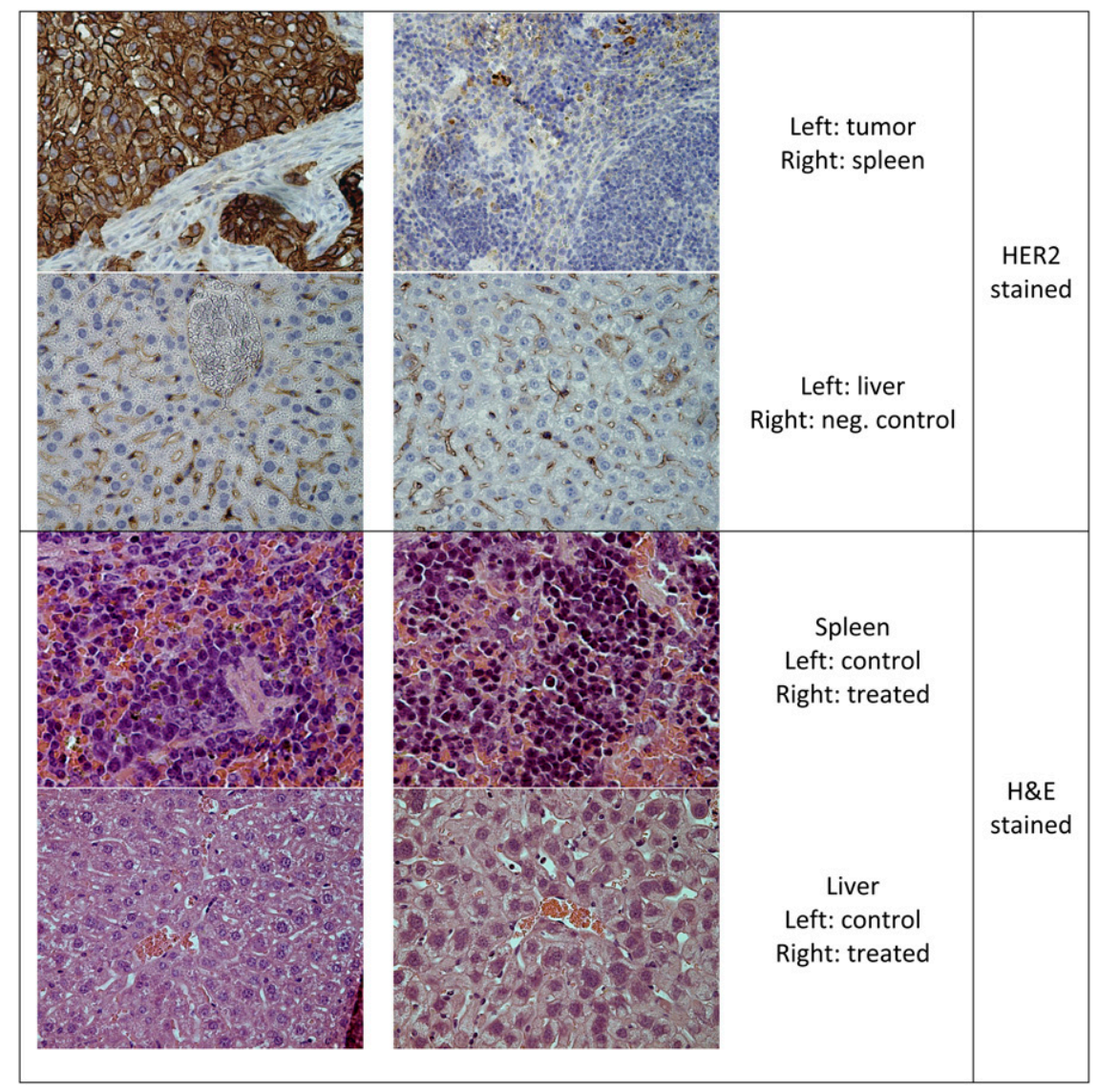

further elevated uptake of targeting liposomes in spleen has been seen $(15,16)$. As revealed by the immunohistochemical analysis of HER2 expression in this study, and as expected, we could not detect HER2 in either liver or spleen whereas tumors were intensively stained. The doses calculated for spleen and liver for the HER2-targeting liposomes were low, taking into account that the effect on tumor cells is dramatic. The highest calculated dose for the spleen was 0.3 Gy, which is expected to be far from radiotoxic levels (17). The lack of HER2 targeting in liver and spleen suggests that although the two-step targeting principle is operative in tumor cells, it likely is not so in liver or spleen. Hence, although uptake in spleen is higher than in tumor cells, this does not necessarily constitute a problem, since the first step in the two-step targeting sequence most probably is nonfunctional.

The primary aim with the strategy based on Nuclisome particles is to eradicate circulating tumor cells in the bloodstream or spread cells in the abdominal cavity. An animal model with circulating tumor cells in the bloodstream is difficult to achieve, and we therefore chose to study the ability of liposomes to target single cells in the abdominal cavity. In the experimental setup, liposomes were injected directly after inoculation of the tumor-cell suspension. Because cells were not allowed to form solid tumors, targeting of single-spread cells was possible. This is a challenging situation since not only one or a few tumors should be treated but instead 10 million cells that each potentially can form metastases. A drawback with this model in comparison with subcutaneous tumors is the problematic scoring of tumor growth. This problem is reflected in the present study, in which 5 animals were censored because of incorrect scoring, that is, not killed by the tumor burden. Although these mice, since they were censored, did not affect the outcome of the study, they affected the significance somewhat.

Clearly, mice that received only vehicle (HEPES buffered saline) were killed first, whereas mice that underwent treatment with the highest radioactive dose survived longest. Statistical analysis of Kaplan-Meier survival plots using the Log-rank test (Mantel-Cox) was done, and there was a significant increase in survival for groups treated with HER2-targeting Nuclisome particles. Already at $0.1 \mathrm{MBq}$ per mouse the survival was prolonged. This is a remarkably low dose, especially in comparison with ${ }^{125}$ I-labeled targeting proteins (18). Survival increased with increasing dose, and at $2 \mathrm{MBq}$ per mouse most of the mice survived the study. Also notable is that more than $50 \%$ of the survivals were tumor-free, compared with the pooled control group of 33 individuals, of which none was tumor free.

One important finding was that treatment with nontargeting liposomes filled with ${ }^{125}$ I-Comp1 did not prolong survival, except somewhat for the highest dose. This observation strengthens the hypothesis that the impressive therapeutic effect of Nuclisome particles is mainly a result of active HER2 targeting. This is noteworthy since previous studies that focused on the administration of F5-targeted liposomes in the blood circulation of subcutaneously xeno- 
grafted mice failed to show enhanced uptake of targeting compared with nontargeting liposomes in tumors $(19,20)$. PEGylated nontargeting liposomes loaded with the cytostatic drug doxorubicin are under clinical evaluation for use as a single agent or in combination with platinum, taxanes, and topotecans for the treatment of ovarian cancer (21). The results of the present study indicate that providing the drug-loaded liposomes with a targeting device might significantly increase efficacy.

In the present study, no acute toxicity from the radiation could be seen in any of the groups. As mentioned, the single organ accumulating the most radioactivity was spleen. However, except for spleen in 1 animal, in which some necrosis was observed, no radiotoxic effect could be seen in any normal mouse organ although there was a clear therapeutic effect on tumor cells. This encouraging finding is most likely explained by the functional two-step targeting of DNA in HER2-expressing cells. It is plausible that the maximum tolerated radioactive dose was not yet reached and a further increase in dose might further improve survival. However, there is a risk that increasing the dose could lead to radiotoxic effects in normal tissues, and careful optimization, including toxicity evaluation, is therefore needed.

\section{CONCLUSION}

The results obtained so far are promising. We showed in this study that mice treated with Nuclisome particles had a significantly prolonged survival and that, when treated with as little as $2 \mathrm{MBq}$ of ${ }^{125} \mathrm{I}$ per mouse, more than half the mice were tumor-free at the end of the study. Importantly, the doses received by normal organs were low. These results encourage further studies, including analysis of the pharmacokinetics and biodistribution of Nuclisome particles in humans. It is in this context noteworthy that our in-house-developed daunorubicin analog allows for simple replacement of ${ }^{125} \mathrm{I}$ with radionuclides suitable for SPECT and PET ( ${ }^{123} \mathrm{I}$ and ${ }^{124} \mathrm{I}$, respectively) to assess normal-tissue doses and tumor-cell specificity in future studies.

\section{DISCLOSURE STATEMENT}

The costs of publication of this article were defrayed in part by the payment of page charges. Therefore, and solely to indicate this fact, this article is hereby marked "advertisement" in accordance with 18 USC section 1734.

\section{ACKNOWLEDGMENTS}

We thank Malin Morin Zetterberg and Dr. Johan Unga for help with liposome production and Dr. Jonny Eriksson for assistance with cryogenic transmission electron microscopy analysis. We further thank Veronika Asplund and Ulrika A Larsson for technical assistance and Prof. Hans Nordgren for support with histologic evaluation. Funding was provided by the Swedish Cancer Society, the Swedish Research
Council, and Stiftelsen Olle Engkvist Byggmästare. Katarina Edwards and Lars Gedda are shareholders of Nuclisome AB. No other potential conflict of interest relevant to this article was reported.

\section{REFERENCES}

1. Hofer KG, Prensky W, Hughes WL. Death and metastatic distribution of tumor cells in mice monitored with ${ }^{125}$ I-iododeoxy-uridine. J Natl Cancer Inst. 1969;43:763773.

2. Kassis AI. The amazing world of Auger electrons. Int J Radiat Biol. 2004;80:789803.

3. Chen P, Wang J, Hope K, et al. Nuclear localizing sequences promote nuclear translocation and enhance the radiotoxicity of the anti-CD33 monoclonal antibody HuM195 labeled with ${ }^{111} \mathrm{In}$ in human myeloid leukemia cells. J Nucl Med. 2006;47:827-836.

4. Costantini DL, Chan C, Cai Z, Vallis KA, Reilly RM. ${ }^{111}$ In-labeled trastuzumab (Herceptin) modified with nuclear localization sequences (NLS): an Auger electronemitting radiotherapeutic agent for HER2/neu-amplified breast cancer. J Nucl Med. 2007;48:1357-1368.

5. Costantini DL, Villani DF, Vallis KA, Reilly RM. Methotrexate, paclitaxel, and doxorubicin radiosensitize HER2-amplified human breast cancer cells to the Auger electron-emitting radiotherapeutic agent ${ }^{111} \mathrm{In}$-NLS-trastuzumab. J Nucl Med. 2010;51:477-483.

6. Ginj M, Hinni K, Tschumi S, Schulz S, Maecke HR. Trifunctional somatostatinbased derivatives designed for targeted radiotherapy using Auger electron emitters. J Nucl Med. 2005;46:2097-2103.

7. Fondell A, Edwards K, Ickenstein LM, Sjoberg S, Carlsson J, Gedda L. Nuclisome: a novel concept for radionuclide therapy using targeting liposomes. Eur J Nucl Med Mol Imaging. 2010;37:114-123.

8. Ickenstein LM, Edwards K, Sjoberg S, Carlsson J, Gedda L. A novel ${ }^{125}$ I-labeled daunorubicin derivative for radionuclide-based cancer therapy. Nucl Med Biol. 2006;33:773-783.

9. Lundqvist H, Stenerlöw B, Gedda L. The Auger effect in molecular targeting therapy. In: Stigbrand T, Carlsson J, Adams GP, eds. Targeted Radionuclide Tumor Therapy. Springer Sciences + Business Media BV; 2008:197-216.

10. Fondell A, Edwards K, Unga J, Kullberg E, Park JW, Gedda L. In vitro evaluation and biodistribution of HER2-targeted liposomes loaded with an ${ }^{125}$ I-labelled DNAintercalator. J Drug Target. 2011;19:846-855.

11. Ghirmai S, Mume E, Tolmachev V, Sjoberg S. Synthesis and radioiodination of some daunorubicin and doxorubicin derivatives. Carbohydr Res. 2005;340:15-24.

12. Chaires JB, Herrera JE, Waring MJ. Preferential binding of daunomycin to 5'ATCG and $5^{\prime}$ ATGC sequences revealed by footprinting titration experiments. Biochemistry. 1990;29:6145-6153.

13. Nielsen UB, Kirpotin DB, Pickering EM, et al. Therapeutic efficacy of anti-ErbB2 immunoliposomes targeted by a phage antibody selected for cellular endocytosis. Biochim Biophys Acta. 2002;1591:109-118.

14. RADAR - available phantoms. RAdiation Dose Assessment Resource Web site. Available at: http://doseinfo-radar.aifm.it/RADARphan.html. Accessed January 27, 2012

15. Brignole C, Marimpietri D, Gambini C, Allen TM, Ponzoni M, Pastorino F. Development of $\mathrm{Fab}^{\prime}$ fragments of anti-GD(2) immunoliposomes entrapping doxorubicin for experimental therapy of human neuroblastoma. Cancer Lett. 2003;197: 199-204.

16. Pastorino F, Brignole C, Di Paolo D, et al. Targeting liposomal chemotherapy via both tumor cell-specific and tumor vasculature-specific ligands potentiates therapeutic efficacy. Cancer Res. 2006;66:10073-10082.

17. Persson M, Gedda L, Lundqvist H, et al. $\left[{ }^{177} \mathrm{Lu}\right]$ pertuzumab: experimental therapy of HER-2-expressing xenografts. Cancer Res. 2007;67:326-331.

18. Santoro L, Boutaleb S, Garambois V, et al. Noninternalizing monoclonal antibodies are suitable candidates for ${ }^{125} \mathrm{I}$ radioimmunotherapy of small-volume peritoneal carcinomatosis. J Nucl Med. 2009;50:2033-2041.

19. Kirpotin DB, Drummond DC, Shao Y, et al. Antibody targeting of long-circulating lipidic nanoparticles does not increase tumor localization but does increase internalization in animal models. Cancer Res. 2006;66:6732-6740.

20. Mamot C, Drummond DC, Noble CO, et al. Epidermal growth factor receptortargeted immunoliposomes significantly enhance the efficacy of multiple anticancer drugs in vivo. Cancer Res. 2005;65:11631-11638.

21. Ferrandina G, Corrado G, Licameli A, et al. Pegylated liposomal doxorubicin in the management of ovarian cancer. Ther Clin Risk Manag. 2010;6:463-483. 